\title{
In search for the lost antibiotic: a multidisciplinary approach to address the problem of the emergence of antibiotic resistance
}

\author{
Giuliana Cangemi', Patrizia Morelli', Maddalena Perotti', Roberto Bandettini', Elio Castagnola², \\ Eugenio Debbia ${ }^{3}$, Giovanni Melioli' \\ I U.O. Laboratorio Centrale di Analisi, Dipartimento di Medicina Sperimentale e di Laboratorio; \\ 2 U.O. Malattie Infettive, Dipartimento di Emato-Oncologia, Istituto Giannina Gaslini, \\ 3 Sezione di Microbiologia, Dipartimento di Scienze Chirurgiche e Diagnostiche Integrate, Università degli Studi di Genova
}

Key words: Antibiotic resistance, Emerging pathogens, New drugs

\section{SUMMARY}

The resistance to antibiotics, appeared in the $50 \mathrm{~s}$, at the same time of the introduction of these drugs, has become today a public health emergency of international importance. This problem is extremely complex because it is based on many factors, including inappropriate use of antibiotics and their massive presence in the feed of farm animals. Today this problem has become a real public health priority not only for the clinical implications (increased morbidity, mortality and duration of disease, the possibility of developing complications and infections), but also for the economic impact of infections by bacteria resistant, due to the additional cost for more expensive drugs and procedures and for stretching the hospitalization. The European Centre for Disease Prevention and Control (ECDPC) in Stockholm has recently estimated that the bacteria with multiple resistances are responsible for about half of thirty-seven thousand deaths per year in the twenty member countries of the European Union. The importance of this phenomenon has prompted several surveillance systems based on the collection of laboratory data to local or national level. To standardize and interpret the data collected and to facilitate comparison between different realities, in 1998 the EU has decided to finance a European surveillance network (EARSS: European Antimicrobial Resistance Surveillance System), which involves several national surveillance networks. Despite the increase of antimicrobial resistance in clinical isolates of both gram positive and gram negative, there are very few new antimicrobial agents in development.

Only a multidisciplinary approach, combining various skills in medicine, can help us cope with this emergency and to use the resources available.

\section{INTRODUCTION}

The resistance to antibiotics, appeared in the $50 \mathrm{~s}$, at the same time of the introduction of these drugs, has become today a public health emergency of international importance.

This problem is extremely complex because it is based on many factors, including inappropriate use of antibiotics and their massive presence in the feed of farm animals. Today this problem has become a real public health priority not only for the clinical implications (increased morbidity, mortality and duration of disease, the possibility of developing complications and infections), but also for the economic impact of infections by bacteria resistant.

In fact this problem is responsible for additional costs for more expensive drugs and procedures and for stretching the hospitalization. The European Centre for Disease Prevention and Control (ECDPC) in Stockholm has recently esti- mated that the bacteria with multiple resistances are responsible for about half of thirty-seven thousand deaths per year in the twenty member countries of the European Union.

The importance of this phenomenon has prompted several surveillance systems based on the collection of laboratory data at local or national level. To standardize and interpret the data collected and to facilitate comparison between different realities, in 1998 the EU has decided to finance a European surveillance network (EARSS: European Antimicrobial Resistance Surveillance System), which involves several national surveillance networks.

Despite the increase of antimicrobial resistance in clinical isolates of both gram positive and gram negative, there are very few new antimicrobial agents in development.

Only a multidisciplinary approach, combining various skills in medicine, can help us cope with this emergency and to use the available resources.

\section{Corresponding author: Corich Lucia}

Università degli Studi di Trieste, Dipartimento di Scienze della Vita

Via L. Giorgieri, I - 34I00 TRIESTE - Tel.: 0405583693 - Fax: 040558369 I

E-mail: luxcor@yahoo.it 


\section{What is antibiotic resistance?}

Antimicrobial resistance is an inevitable evolutionary response to antibiotic therapy.

Significant resistance to penicillin appeared in Staphylococcus aureus just one year after the introduction of penicillin resistance and multiple strains have appeared in the hospitals a few years later.

Since the introduction of fluoroquinolones in clinical practice in the 80 's resistance to these compounds has become common in some bacterial species, especially in nosocomial strains of staphylococci, Enterobacteriaceae and Pseudomonas aeruginosa. The recent isolation of nosocomial strains of $S$. aureus vancomycin-resistant is the latest in a chain of events that have antibiotic resistance one of the major health problems of global significance.

At the origin of the onset of acquired resistance, there are two fundamental genetic mechanisms:

a) mutation and selection;

b) exchange and acquisition of resistance genes between strains and species.

The first mechanism is of "Darwinian" type: within a sensitive bacterial population, the resistant bacterial cell survives the selective pressure exerted by the antibiotic and by duplication it will gives rise to a resistant population. The resistance is due to a mutation and will be transmitted "vertically" to the clone. In the second mechanism, the transmission of genetic information linked to the resistance is "horizontal": susceptible strains receive genes from resistant strains.

A phenotypic antibiotic resistance in a strain may occur with different biochemical mechanisms: inactivation of the antibiotic, alteration of the target structure, expression of an alternative target, and changes in the permeability.

At the base of the first mechanism there is the synthesis of enzymes that inactivate antibiotics.

- This mechanism is typical of the resistance to $\beta$ lactamics by $S$. aureus. B-lactamases hydrolyze the B-lactam ring of penicillin. Among the Gram negatives there are enzymes capable of destroying the cephalosporins and monobactams of the third generation: these enzymes are called extendedspectrum ß-lactamase (ESBL).

Other B-lactamase, defined carbapenemase, are expressed at low levels in Gram negative and are active on carbapenems.

The mechanism of resistance that is at the basis of the methicillin-resistance consists in the alteration of the target structure. In $S$. aureus such resistance is encoded by the expression of the mecA gene, which produces a Penicillin Binding Protein (PBP) 2a, of modified type, which has a very low affinity for penicillins.
Normally these resistances are expressed in strains isolated in nosocomial environment, however, community acquired methicillin-resistant strains are becoming more frequent, which are even more virulent because of the production of a typical toxin.

Resistance to glycopeptides (vancomycin and teicoplanin) is mediated by mobile elements of DNA (plasmids, transposons) among the grampositive bacteria.

This resistance was first described in 1986 in Great Britain and since then it has spread around the world in a polyclonal way because of the ease of enterococci to transfer and take such genetic elements. In fact they also passed to S. aureus leading to the emergence of strains with reduced susceptibility to glycopeptides (VISA, VRSA).

All resistances described so far can be coexpressed, resulting in a multidrug resistant phenotype (MDR), i.e. resistant to at least three of the following classes of antibiotic drugs: beta-lactams, carbapenems, aminoglycosides and fluoroquinolones (5).

\section{Why are there no new antibiotics on the market?}

The development of new antibiotics is influenced by several factors including the need for substantial funds for research, marketing strategies that can address the development towards more profitable pharmaceutical sectors and complexity of clinical trials.

These problems discourage the pharmaceutical industry in developing innovative antimicrobial molecules and limit the production to drugs chemically similar to those already in distribution.

Although some antimicrobials are at an advanced stage of development for the treatment of infections caused by major pathogens such as $S$. aureus methicillin-resistant (MRSA), a few molecules have been proposed for the treatment of pathogens such as Enterococcus, Klebsiella, Acinetobacter, Pseudomonas and Enterobacter. Up to now for the treatment of infections by pathogenic Gram negative MDR, such as Acinetobacter baumannii and Pseudomonas aeruginosa, there is no drug that has reached an advanced state of development, therefore it was necessary to re-use antibiotics now fallen into disuse such as colistin $(1,4)$.

\section{What is the role of the clinical pathology laboratory?}

As regards the diagnostic laboratory testing phenotypic drug susceptibility and identification of bacteria are carried out using standardized and 
automated methods. The susceptibility (i.e. the in vitro evaluation of bacterial sensitivity to antibiotics) is defined by determining the value of Minimum Inhibitory Concentration (MIC) of the tested drugs. The results of these values are interpreted (sensitive or resistant) according to the provisions of the European Committee on Antimicrobial Susceptibility System Testing (EUCAST) and by using an expert system that guides the microbiologist in the interpretation of the test and may suggest additional tests to confirm the highlighted resistances.

As regards the impact of resistance on blood cultures submitted to the Clinical Pathology Laboratory of G. Gaslini Institute, from 2005 to 2010, 83 isolates of $S$. aureus (15 MRSA), 242 Gram negative (1 MDR and 7 ESBL producers) out of 1945 patients with positive blood culture have been reported. There were no vancomycinresistant enterococci and staphylococci.

To monitor the colonization of MRSA in critical departments (such as resuscitation, at risk infants, hematology-oncology) and patients transferred from other hospitals, strategies based on molecular biology techniques have been implemented for the detection of the gene for methicillin-resistance (mecA).

Analysis of the data obtained so far demonstrate an incidence of MRSA by $6 \%$. Although the percentage is far below the world average (which exceeds $50 \%$ with variations from country to country) we can not lower our guard and we must implement all the laboratory strategies currently available to meet this emergency.

\section{What are the future diagnostic and therapeutic scenarios?}

Since no new antibiotics are being introduced in the short time, we need to explore models of prevention, to increase the use of rapid diagnostic tests, and to implement knowledge on pharmacokinetic and pharmaco-dynamics.

And here is that the lost drug is really lost, and his research can only go through a scientific and organizational model that will ship us to the time of the availability of new drugs.

Today the ideal antibiotic to treat bacterial infections is a molecule already in use, but used in a new, highly integrated way where no detail is left to chance. In practice it is necessary to preside over many different fields of medicine to achieve this result.

With regard to the response of the laboratory strategies to be adopted are: - a rapid diagnosis (antigen detection, nucleic acid detection), which also includes the demonstration of genes and / or proteins associated with bacterial resistance. The technology "next generation sequencing" applied to bacterial genomics will surely be of great impact in this field (2).

In this way an empirical therapy, based only on the knowledge of the spectra of sensitivity of a particular microbe, would be markedly improved - the assessment of the actual capabilities of an antibiotic to kill a microbe. Indeed we are accustomed to speak of sensitivity and resistance to an antibiotic, but this is a very academic approach and away from reality. Currently, the concentrations of different antibiotics occurring to kill many bacteria are well known.

We could almost speak of reference values as for any other blood chemistry parameter. Suppose, for example, that 100 different strains of a microbe have a threshold of 50 units of antibiotic above which are killed. It is intuitive that a concentration of antibiotic achieved in the tissue, very close to the threshold of resistance (for example 60 units) will have a very different significance from a concentration that is far from the threshold (for example 600 units).

In the first case we can imagine that it can take a little to get a full resistance, while in the second case the situation may be more easily manageable. Furthermore it is evident that in the case of a resistant strain the possibility of reaching concentrations close to that required into the tissues, could lead us to use different strategies (site regional infusion?) to overcome the threshold of resistance. But which is the concentration of antibiotic that reaches the target tissue?

Clinical pharmacology, together with analytical chemistry, allows documenting the presence of a drug in a biological matrix, allowing a highly specific identification and quantification.

The introduction of methods of mass spectrometry associated with liquid chromatography (LCMS/MS) is essential in order to guard this field. This is a new challenge for laboratory medicine that as it's capable of measuring the levels of digoxin or immunosuppressive drugs, should organize itself to measure the levels of antibiotics and antifungals to face the emergency in progress. The treatment of infections, especially in the presence of multiresistant bacteria, is a delicate balance between the availability of the drug at the required concentration in a given district, the sensitivity of the microorganism to the molecule and the general toxicity and/or local antibiotic administered.

The infectious disease specialist, pharmacologist, microbiologist and toxicologist will work in great synergy to win this battle (3).

Surely we have the means, we must make progress in the organization. 


\section{BIBLIOGRAFIA}

1. Boucher HW, et al. Bad bugs no drugs: no ESKAPE! An update from the Infectious Diseases Society of America. Clinical Infectious Diseases, 2009; 48:1-12

2. MacLean D, Jones JDG, David J. Application of 'next-generation' sequencing technologies to microbial genetics. Nature Reviews Microbiology 2009; 7: 287-96.
3. Melioli G. La ricerca clinica in popolazioni particolari. In: Alla ricerca del farmaco perduto di Carlo Tomino, 2011: cap.9: 271-91.

4. Theuretzbacher U. Future antibiotics scenario: is the tide starting to run? International Journal of Antimicrobial agents 2009; 34: 15-20.

5. Watson R. Multidrug resistance responsible for half of deaths from healthcare associated infections in Europe. British Medical Journal. 2008; June 7: 1266-7. 